\title{
Investigador novel: estrategias y herramientas en el mundo conectado
}

\author{
Researcher Novel: Strategies and Tools in the connected world \\ Víctor González Calatayud \\ Universidad de Murcia \\ Victor.gonzalez@um.es \\ María del Mar Román García \\ Universidad de Murcia \\ Mariamar.roman@um.es
}

Recibido: $27 / 06 / 2016$

Aceptado: 30/06/2016

Publicado: 30/06/2016

\section{RESUMEN}

El momento en el que un estudiante comienza con el proceso investigador, ya sea en un trabajo fin de master o una tesis, se encuentra en muchas ocasiones desbordado por la gran cantidad de cosas nuevas que debe manejar en poco tiempo. Para este momento existen una serie de herramientas que nos permiten regular todo el proceso, siendo de interés para el propio investigador algunos enfoques como el de Entorno Personal de Aprendizaje. De este modo, nos encontramos con herramientas que nos van a permitir realizar una buena búsqueda de información en las bases de datos, herramientas que facilitan la gestión de la abundante información que encontraremos en dicho proceso así como programas estadísticos -tanto cuantitativos como cualitativos- que van a hacer que el proceso de análisis de datos sea algo menos complicado.

\section{PALABRAS CLAVE}

Investigación; TIC; gestión; proceso

\section{ABSTRACT}

When a student starts with the research process, either in a final work of master or thesis, it is often overwhelmed by the large number of new things to handle in a short time. For this process there are a number of tools that allow us to regulate the whole process, being of interest to the researcher some approaches such as Personal Learning Environment. Thus, we find tools that will allow us to make a good search for information in databases, tools that facilitate the management of the abundant information that we find in the process as well as statistical programs -both quantitative and qualitative-that make the process of analysing data less complicated.

\section{KEYWORDS}

Research; ICT; management; process

\section{CITA RECOMENDADA}

González, V. y Román, M. (2016). Investigador novel: estrategias y herramientas en un mundo conectado. RIITE. Revista Interuniversitaria de Investigación en Tecnología Educativa, 0, 95-108. Doi: http://dx.doi.org/10.6018/riite/2016/262241 


\section{INTRODUCCIÓN}

Escribir este artículo ha supuesto para nosotros el reto de enfrentarnos a revivir los primeros pasos dubitativos en los que la mayoría de estudiantes se encuentran al iniciar cualquier proyecto de investigación, ese momento inicial en el que la única certeza parece ser que será un camino lleno de incertidumbres.

¿Pero qué podemos aportar nosotros a los futuros estudiantes que tengan que realizar un proyecto de investigación? Como investigadores noveles lo que queremos es que aquellas personas que tengan que comenzar un proyecto de investigación encuentren en estas líneas algunas palabras de aliento, necesario en algunos instantes de dudas, como algunas herramientas que pueden sernos muy útiles en determinados momentos de nuestra investigación.

Somos conscientes que no hay recetas mágicas y que cada experiencia de investigación, desde los recientes Trabajos Fin de Grado (en delante TFG), los Trabajos Fin de Master (TFM) hasta la Tesis, como acción individual que es, se convierte en un proceso de aprendizaje único e irrepetible.

Buscamos transmitir como para nosotros, ese proceso de elaboración de un proyecto de investigación, se ha convertido en una experiencia de aprendizaje, ese tipo de experiencias de las que nos habla Jorge Larrosa (2007) algo que no se puede transmitir mecánicamente, que a nadie se le puede ahorrar, algo que nos forma y nos transforma.

Entendemos que investigar es "obtener nuevos conocimientos sobre el mundo que nos rodea, entender lo que permanecía confuso o escondido" (Sabino, 1998) y para ello necesitamos tener algunos conocimientos sólidos sobre lo que vamos a investigar, lo que ya conocemos, y lo que la ciencia nos está diciendo al respecto, por eso el proceso de investigación resulta ser un proceso laborioso y exhaustivo y con el que se espera, tanto si hablamos de Tesis como de otros trabajos de investigación (TFM y TFG) que aportemos algo nuevo al conocimiento ya existente, además de certificar que el estudiante tiene, en los distintos niveles a los que hacemos mención, esa competencia investigadora.

Tampoco olvidamos que este proceso es creativo, no se trata de leer y escribir, se trata de crear nuevo conocimiento, y eso requiere dar en muchas ocasiones pasos atrás, para probar nuevos caminos e incluso repetir procesos, pero con distintas miradas.

\section{INVESTIGAR HOY}

Los cambios en la sociedad a día de hoy con respecto a los avances tecnológicos es una cuestión indiscutible y palpable en muchos ámbitos de nuestra vida. La forma en que nos comunicamos, en la que compramos, nos relacionamos e incluso aprendemos ha sufrido cambios, por lo que los procesos de investigación también están sujetos a esta dinámica de cambio. ¿Pero se trata solamente de una cuestión de herramientas tecnológicas? como afirma Formanchuck (2013) además del uso de las tecnologías es necesario un cambio de actitud basada en una filosofía 2.0.

Como exponen Estalella y Ardevol (2011), en las ciencias naturales y de la salud la e-sciencie ha aparecido como forma de conjugar las prácticas de investigación, las formas organizacionales de la ciencia junto con la convergencia de tres tecnologías: redes de comunicación a gran escala, acceso a grandes bases de datos y el acceso a la computación distribuida (Wouters, 2006). Pero desde las ciencias sociales parece tener, aún a día de hoy, un menor índice de absorción.

Entendemos que la incorporación de tecnologías digitales en los procesos de investigación debería tratarse como una cuestión transversal, comenzando por la formación de los futuros 
investigadores. No se trata de una mera asimilación de herramientas en dichos procesos sino de la asimilación de la e-research como punto de anclaje.

Partimos del concepto de Wouters, Beaullieu y Beaulieu y Wouters $(2006,2008)$ que indican que la e-research es un proceso de incorporación progresiva de las tecnologías en la producción de conocimiento de las ciencias sociales, poniendo el acento en tres importantes cuestiones:

- Tiene una dimensión transversal, ya que no afecta solamente a una sola disciplina, sino que involucra a todas las ciencias sociales y no solamente a tecnologías digitales.

- Está incorporación implica un cambio en las prácticas de los investigadores.

- Estas tecnologías hacen posible aumentar, intensificar las prácticas de investigación (de ahí la "e" de "enhanced"). Haciendo referencia a esa intensificación en el aumento en el acceso a la información, en las posibilidades de colaboración y en la producción de datos.

Este concepto de e-research trasciende, como bien apuntan Sánchez y Teruel (2013) al concepto de e-sciencies ya que en el ámbito desde el que estamos escribiendo, las Ciencias Sociales, nos parece más inclusivo, porque nos ayuda a poder transformar las prácticas de producción de conocimiento, ayudándonos en el reto metodológico que todo esto supone. Pero más allá de las necesarias cuestiones de reflexión en torno a la adopción de esta nueva cultura de investigación 2.0, debemos ser prácticos y saber que, en cada una de las fases de la investigación, comenzando por la búsqueda y gestión de la información hasta la fase de difusión de resultados, podemos contar con aplicaciones, herramientas y estrategias propias de la web social que nos pueden facilitar la realización de ese proyecto de investigación. Además de contribuir en otros aspectos como el aumento de nuestra red de colaboración, la construcción de nuestra identidad digital y la aportación al movimiento de Open Content.

Asumir esta filosofía también supone reconocer la necesidad de que tanto los actuales profesionales de la investigación como los futuros investigadores desarrollen ciertas competencias digitales, lo que supone ir más allá del uso instrumental de las mismas (Gutiérrez, 2011).

Un enfoque que puede resultar muy esclarecedor para afrontar esta nueva cultura 2.0 en la investigación es el de entornos personales de aprendizaje (PLE por sus siglas en inglés). Este enfoque nos ayudará en nuestro proceso de aprendizaje que supone llevar a cabo una Tesis, TFM o TFG, ya que se requiere una alta dosis de autonomía y de responsabilidad por parte del aprendiz.

Siguiendo la definición de Castañeda y Adell (2013, p. 15) "el PLE de las personas se configura por los procesos, experiencias y estrategias que el aprendiz puede, y debe, poner en marcha para aprender (...) y está determinado por las posibilidades que las tecnologías abren y potencian". La adopción y desarrollo de este enfoque nos lleva también a la idea de investigación aumentada "aquella investigación enriquecida con procesos y herramientas que tienen como fin una mejor gestión del conocimiento" (Peña, 2013, p. 95). La unión de estos dos conceptos puede enriquecer nuestro proceso de investigación, aumentando el potencial de cada uno de los pasos que llevaremos a cabo y dotando a nuestro proyecto de una mayor transparencia. En la siguiente tabla podemos encontrar ejemplos de esa investigación aumentada a la que hacemos referencia, siguiendo una línea temporal de dicho proceso. 
Tabla 1 investigación aumentada en la línea del tiempo de una investigación (op.cit)

\begin{tabular}{ll}
\hline \multicolumn{1}{c}{ Línea de tiempo de una investigación } & \multicolumn{1}{c}{ Investigación aumentada } \\
\hline Idea de investigación & Página personal o del proyecto \\
\hline \multirow{2}{*}{ Estado de la cuestión } & Lector de feeds, RSS, micro blog \\
& Listas de correo Alertas \\
& Plataformas de redes sociales \\
\hline Revisión de la literatura & Repositorios abiertos Publicaciones en abierto \\
\hline Asistir a un evento (p.ej. congreso) & Presentaciones del evento. Vídeos del evento \\
\hline & Seguir el evento vía micro blog \\
Notas de campo & Marcadores sociales \\
& Compartidos en redes sociales \\
& Blog \\
\hline Working paper & Wiki \\
& Gestor bibliográfico \\
Comunicación en evento académico & Repositorio personal de datos en abierto \\
& Auto-archivo (self-archiving) Comentarios en blogs \\
& Blog \\
& Auto-archivo \\
Artículo académico & Presentación de la comunicación. Podcast \\
& Vidcast \\
\hline Consolidación de red académica & Plataformas de redes sociales Microblog \\
Exploración de una nueva investigación & Blog \\
\hline
\end{tabular}

Otro análisis muy interesante es el que apuntan Rodera y González (2014) en su artículo Tesis 2.0: herramientas para su diseño, desarrollo, lectura y difusión, dónde podemos encontrar diferentes herramientas que nos pueden ayudar en las distintas fases de realización de la Tesis. En este caso poniendo el foco en las posibilidades que las propias herramientas nos ofrecen.

\section{RECURSOS PARA LA ORGANIZACIÓN DEL PROCESO INVESTIGADOR}

Es totalmente cierto que durante mucho tiempo ha sido costumbre hacer uso de nuevos desarrollos tecnológicos para aliviar la carga de tareas complejas o de tareas rutinarias. Lo cual ha repercutido tanto en la investigación como en cualquier aspecto de la actividad humana (Roberts y Wilson, 2002). En el campo de la investigación se evidencia, por ejemplo, en las consultas que los investigadores hacen en diversos buscadores o en la participación en foros de investigación (Corredor Acosta y Sandino León, 2009). Sin embargo, este desarrollo tecnológico, más concretamente la digitalización de la información y la facilidad de editar y publicarla en la red, dificulta el proceso de investigación al existir una ingente cantidad de información a tener en cuenta en nuestra investigación.

Por esta razón, se hace imprescindible conocer todas las herramientas que existen y que nos pueden facilitar todo el proceso, sobre todo para aquellos que están empezando, en lo concerniente a la búsqueda de información, cómo gestionarla o dónde publicar. Si una herramienta que está disponible nos ayuda a reducir tiempo o a mejorar el trabajo, porque no utilizarla (Roberts y Wilson, 2002). De este modo, las TIC nos van a facilitar el proceso de búsqueda al disponer de bases de datos donde podemos encontrar artículos de investigación, libros digitales, tesis, actas de congresos, etc. Una vez que tenemos esa información, con ciertas herramientas podremos gestionar toda esa información sin la necesidad de usar incluso su propio ordenador. Por ejemplo, tenemos herramientas como Dropbox o Google Drive que nos van a permitir guardar en la nube y compartir con quien lo necesitemos. También tenemos herramientas para gestionar directamente la bibliografía que ayudan a simplificar la tarea de referenciar, como son Mendeley, Ednote o Zotero. 
Asimismo, tenemos otras herramientas, tanto sincrónicas como asincrónicas que nos van a permitir llevar a cabo un trabajo colaborativo dentro del proceso (Arbeláez Gómez, 2014).

\subsection{Búsqueda Bibliográfica: Técnicas y herramientas.}

Teniendo en cuenta el contexto en el que nos encontramos, la adecuada gestión del conocimiento se convierte en un objetivo primordial para la actualización de cualquier profesional. Para llevar a cabo esa adecuada gestión es necesario acudir a buscadores de información, programas informáticos alojados en la red. Para realizar ésta búsqueda requiere de un proceso sistemático que implica diversos pasos, empezando por identificar claramente el ámbito donde se genera la duda para pasar a elaborar la pregunta de investigación. Además, se necesita de una selección adecuada de la herramienta de búsqueda a utilizar para que la misma nos sirva para la toma de decisiones (Del Cura González y Sánchez-Celaya del Pozo, 2007).

Para realizar una búsqueda sistemática fiable y reproducible encontramos varios protocolos o métodos a seguir. Principalmente han sido desarrollados en el campo de la salud, pero cada vez se están realizando en otros ámbitos como son las ciencias sociales. Las revisiones sistemáticas son investigaciones científicas en las que la unidad de análisis son los estudios originales primarios, con los que se pretende contestar a la pregunta de investigación formulada. Por el contrario, a las revisiones que no siguen un proceso sistemático, las cuales son denominadas como revisiones narrativas, no se les considera un proceso formal de investigación, sino más bien un formato de literatura científica basada principalmente en opinión (Ferreira González, Urrútia, y Alonso-Coello, 2011). Las revisiones sistemáticas van a limitar el sesgo y el error aleatorio mediante estrategias que incluyen: la búsqueda sistemática y exhaustiva de lo más relevante; la selección, mediante criterios específicos y reproducibles, de los artículos a incluir; y la descripción del diseño de los estudios originales, su síntesis e interpretación de los resultados.

La declaración QUOROM (Quality of Reporting of Meta-analysis) fue una de las primeras en establecer unas normas para mejorar la calidad de las revisiones sistemáticas y, por ende, de los metaanálisis. Esta declaración incluye 18 ítems que los editores y autores deberían considerar a la hora de publicar su trabajo, además de incluir un diagrama de flujo que describe todo el trabajo. Después de la revisión de la misma, en 2009 surgió la declaración PRISMA (Preferred Reporting Items for Systematic reviews and Meta-Analysis), en la cual se establecen 27 ítems que se deberían incluir con la debida explicación del mismo (Urrútia y Bonfill, 2010).

Dentro de estas normas es muy importante establecer que bases de datos son utilizadas para la búsqueda y justificar por qué esas y no otras. Estas bases de datos son las principales herramientas que nos vamos a encontrar para la búsqueda de bibliografía. Son muchas las bases las que existen y la posibilidad de utilizarlas va a depender fundamentalmente de si la universidad a la que pertenecemos tiene o no acceso a las mismas. La importancia de estas varía según en el ámbito en el que nos movamos. Así, por ejemplo, en el campo de la salud PubMed es de las más importantes. En muchos casos para poder acceder a estas bases de datos necesitamos "estar" en la universidad. Podemos estar físicamente, por lo que utilizamos directamente la IP de la universidad, o podemos utilizar alguno de los métodos que la mayoría de universidades tienen para que los investigadores tengan acceso desde fuera de la universidad. En algunos casos se utiliza un servidor proxy o en otros casos una aplicación como puede ser un escritorio virtual.

Para realizar la recopilación de documentos primarios podemos acudir a bases de datos generalistas o temáticas. En el campo de la educación y, por ende, de la tecnología educativa las bases de datos con más referencias y más utilizadas son la Web of Science y Scopus. La Web of Science pertenece a Thomson Reuters, la cual es la que elabora también el Journal Cititation Reports (JCR). Por su parte, Scopus pertenece a Elsevier, una de las compañías más importantes en cuanto a revistas científicas se refiere. Ambas bases de datos permiten realizar una búsqueda muy concreta estableciendo si queremos buscar los términos en el título, resumen y palabras clave, por el/los autor/es o incluso por el DOI (Digital Object Identifier). También podemos realizar 
una búsqueda en unos años concretos, establecer qué tipo de documentos queremos buscar (artículo, libro, revisión, etc.) y el área concreta.

Scopus

Scopus SciVal | Victor González v Logout Help •

\begin{tabular}{|l|l|l|l|}
\hline Search Alerts & Lists & My Scopus \\
\hline
\end{tabular}

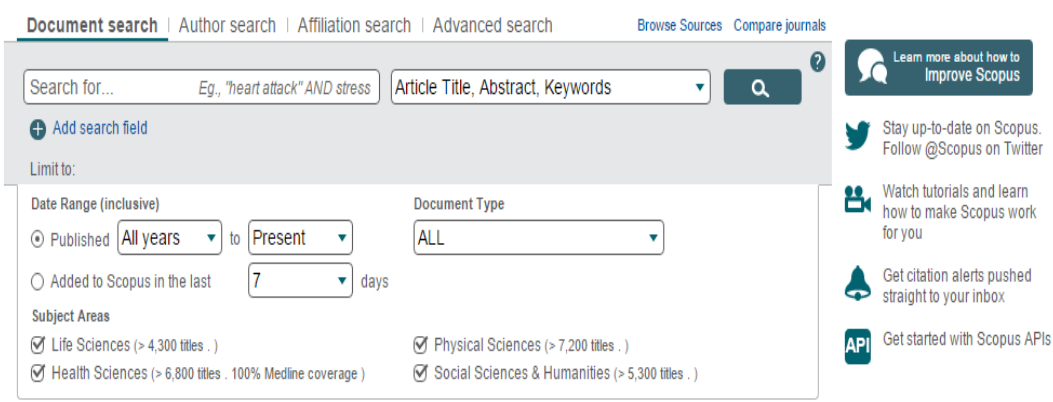

Figura 1: Página principal de la base de datos Scopus

Para que la búsqueda en la base de datos tenga éxito requiere que las palabras utilizadas sean las adecuadas y conocer las técnicas de búsqueda. Normalmente, las búsquedas se realizan a través de palabras clave o por descriptores. Como indican Sureda, Comas, Oliver, y Guerrero (2010), es conveniente en primer momento identificar los conceptos o palabras clave que mejor definen la problemática. En segundo lugar, utilizar sinónimos y traducir esas palabras a otros idiomas, especialmente al inglés, así como consultar tesauros ${ }^{1}$. Por último, estos autores indican que se deben utilizar los operadores booleanos²: OR, AND y NOT. OR se utiliza cuando queremos información de dos temas o términos distintos, por lo que se amplia y diversifica la búsqueda. AND es un operador de intersección que nos dará como resultado solo aquellos documentos que incluyan ambos términos. Y NOT es un operador de exclusión, el cual nos ayuda a excluir aquello que no queremos. Otro de los aspectos a la hora de buscar son los truncamientos (Sureda et al., 2010). Los mismos nos permiten buscar a partir de la raíz de la palabra utilizando el símbolo *.

Por último, cabe destacar algunos portales web, las cuales se les ha nombrado como redes sociales científicas, las cuales permiten a los investigadores estar conectados con el resto de investigadores e intercambiar información sobre su campo de investigación. El punto fuerte de estos portales es que son gratuitos, permiten compartir los trabajos de investigación con facilidad y solamente necesitas tener un perfil en las mismas. Las dos que actualmente más son utilizadas son ResearchGate.net y Academia.edu. Ambas apuestan por el acceso abierto de la ciencia.

\subsection{Gestión Bibliográfica}

Como ya se ha hecho mención, la cantidad de información que podemos recuperar es abrumadora. Incluso con la utilización de técnicas de revisión sistemática la cantidad de documentos que podemos tener hacen que la tarea de gestionar todos esos documentos sea bastante complicada. Para solucionar o reducir este problema surgen los conocidos como gestores bibliográficos.

\footnotetext{
${ }^{1}$ Un tesauro es un vocabulario controlado que se aplica para indexar y recuperar información en una base de datos.

${ }^{2}$ Conjunto de técnicas para expresar operaciones de conjunto utilizadas normalmente en el campo de las matemáticas.
} 
Son programas que nos permiten almacenar las referencias obtenidas durante la búsqueda para su posterior gestión. En algunos casos se requiere de la instalación de un programa en el ordenador, mientras que en otras ocasiones se accede a través de Internet. Tienen principalmente dos partes: una que nos permite administrar las referencias bibliográficas que se añaden y, por otro, la que nos permite insertar citas de esas referencias en un documento mediante una herramienta interrelacionada con el procesador de textos (Duarte-García, 2007). Podemos encontrar muchos programas de estas características, aunque los que más se usan actualmente son Mendeley, Zotero y Ednote (figura 2). Mendeley y Ednote son aplicaciones con propietario, la primera pertenece a Elsevier mientras que la segunda a Thomson Reuters, aunque en ambos casos su uso es gratuito. Por su parte, Zotero es una aplicación libre y abierta desarrollada por el Centre for History and New Media de la George Maison Univeristy.

What tools/sites do you use for reference management?

882 out of 1000 people answered this question

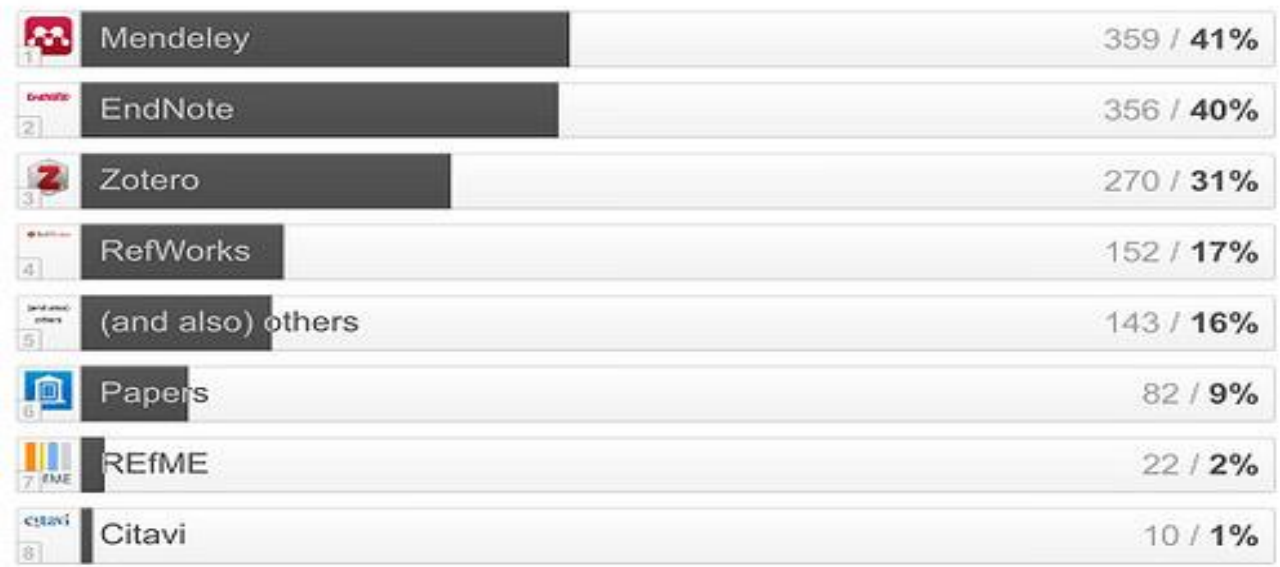

Figura 2: Gestores más utilizados por los investigadores recogido de Fenner, Scheliga, y Bartling, (2014)

Todos estos gestores se utilizan de forma bastante similar y permiten realizar acciones muy diversas en relación a la gestión de información. Así, en general, permiten: crear y gestionar bases de datos; importar automáticamente referencias desde otras bases; y crear bibliografía y citaciones con distintos estilos en un documento. Además, permiten en algunos casos crear grupos colaborativos con los que compartir referencias sobre un campo concreto (Fenner et al., 2014). Algunos de estos gestores permiten igualmente añadir documentos PDF de texto completo y trabajar dentro de la aplicación con ellos. Todos los gestores permiten introducir manualmente los datos bibliográficos, pero es más conveniente y seguro extraerlos de las bases de datos de forma automática. Asimismo, permiten exportar las referencias en formato BibTeX RIS, lo cual facilita que se puedan compartir dichas referencias con los compañeros, aunque estos utilicen otro gestor.

Por último, aunque ya hemos hecho mención, tenemos los conocidos Digital Object Identifier (DOI) que nos ayudan a gestionar adecuadamente las referencias. En la mayoría de revistas se utilizan los DOI para identificar la revista y cada uno de sus artículos publicados. Estos DOI son emitidos por CrossRef, una organización sin ánimo de lucro. Los DOI también se utilizan con actas de congresos y capítulos de libro. Con los DOI los gestores bibliográficos pueden utilizarlos para extraer toda la información necesaria para citar el trabajo en sí, el cual puede ser obtenido de forma sencilla en la página de CrossRef $^{3}$.

${ }^{3}$ La página para buscar el DOI: http://www.crossref.org/guestquery/ 


\subsection{Publicaciones científicas: dónde publicar}

En los últimos 25 años se ha producido un cambio en la evaluación del impacto de la investigación, pasando fundamentalmente a una evaluación por pares a otra cuantitativa a través del análisis de las citas de artículos de revistas. Este hecho se ha visto incrementado gracias al paso de las publicaciones electrónicas (Fenner, 2014). Conocer el impacto que tienen las revistas, por tanto, se ha convertido en uno de los aspectos importantes que los investigadores deben de conocer para publicar y que su investigación llegue a más investigadores. Tampoco podemos olvidar que la evaluación de la actividad investigadora del profesorado se realiza en parte con el índice de impacto y cuartil de las revistas donde se ha publicado, así como las bases de datos donde están indexadas.

El factor de impacto es uno de los indicadores bibliométricos que más se utilizan. Este mide la repercusión que ha tenido una revista a partir del análisis de las citaciones que recibieron los artículos publicados en la misma revista. Con este dato se pueden comparar revistas, establecer rankings y reflejar la relevancia (Universidad Autónoma de Barcelona, 2009). Suele ser calculado mediante la división del número total de citas obtenidas en un año por el número de artículos publicados en esa revista en dos o tres años. A nivel internacional destacan dos fundamentalmente, el proporcionado por Journal Citation Report (JCR) y Scimago Journal Rank (SJR).

El índice de impacto del JCR analiza a más de 7000 títulos, pero, en contrapartida, hay pocas revistas de lengua no inglesa. Para buscar el factor de impacto de una revista en el mismo solo necesitamos acudir a la página de la Web of Science y en la barra de herramientas de la parte superior encontraremos el enlace para ir al mismo. Desde la página que se nos abre (figura 3) podemos ver el ranking de las revistas completo o reducirlo a un campo temático concreto.

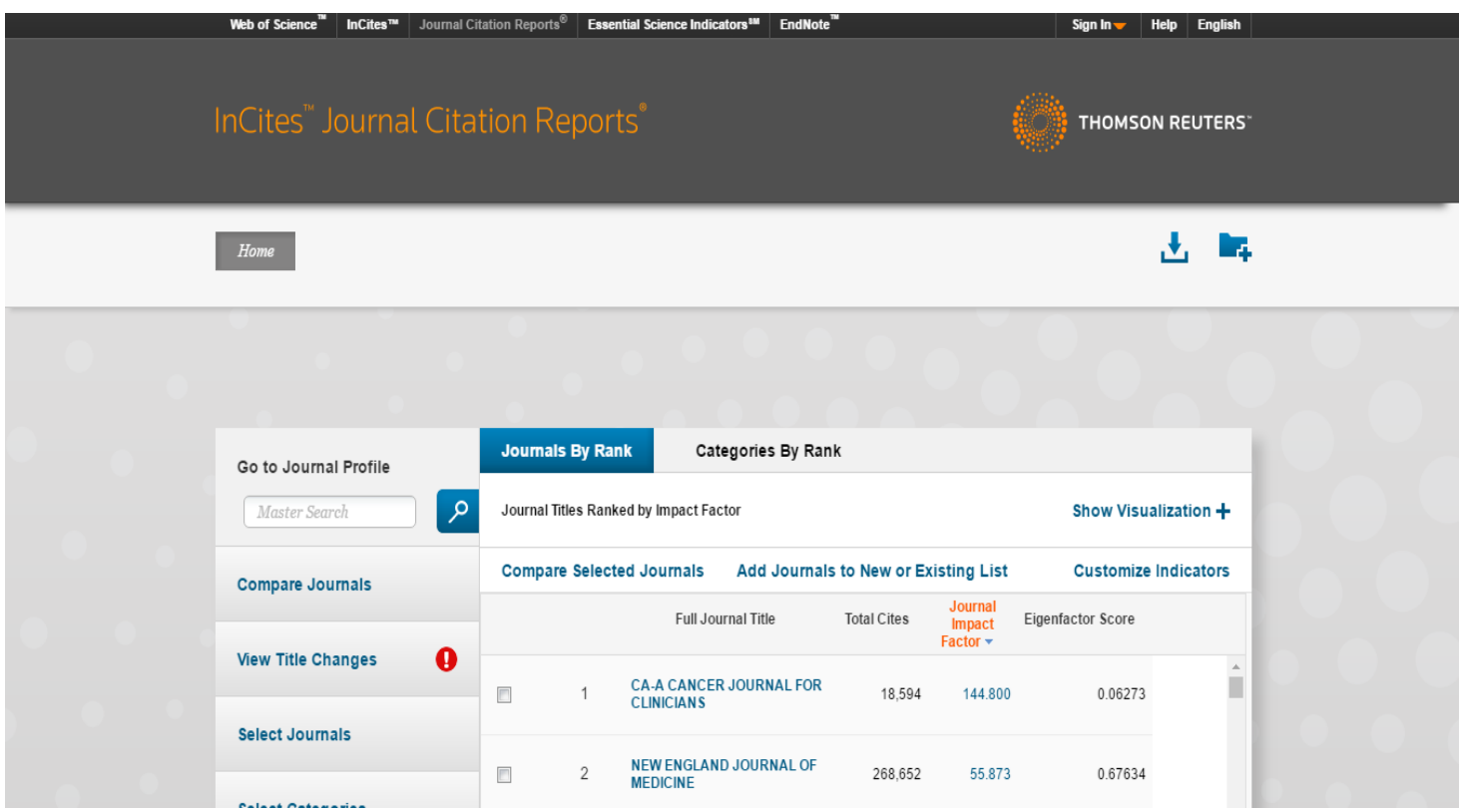

Figura 3: Página web Journal Citation Report

Por su parte, SJR calcula el factor de impacto basándose en la información incluida en su base de datos Scopus. A diferencia del JCR, aquí se combina el número de citas con la influencia de las publicaciones que la citan, por lo que las citan no valen lo mismo. Para acceder tenemos que acudir a la página www.scimagojr.com. Cuando buscamos una revista concreta nos indica en qué áreas se incluye y en qué cuartil se encuentra. También nos permite comparar revistas, categorías y/o países. Además, cuando nos encontramos en la base de datos Scopus podemos encontrar el SJR de las revistas. 


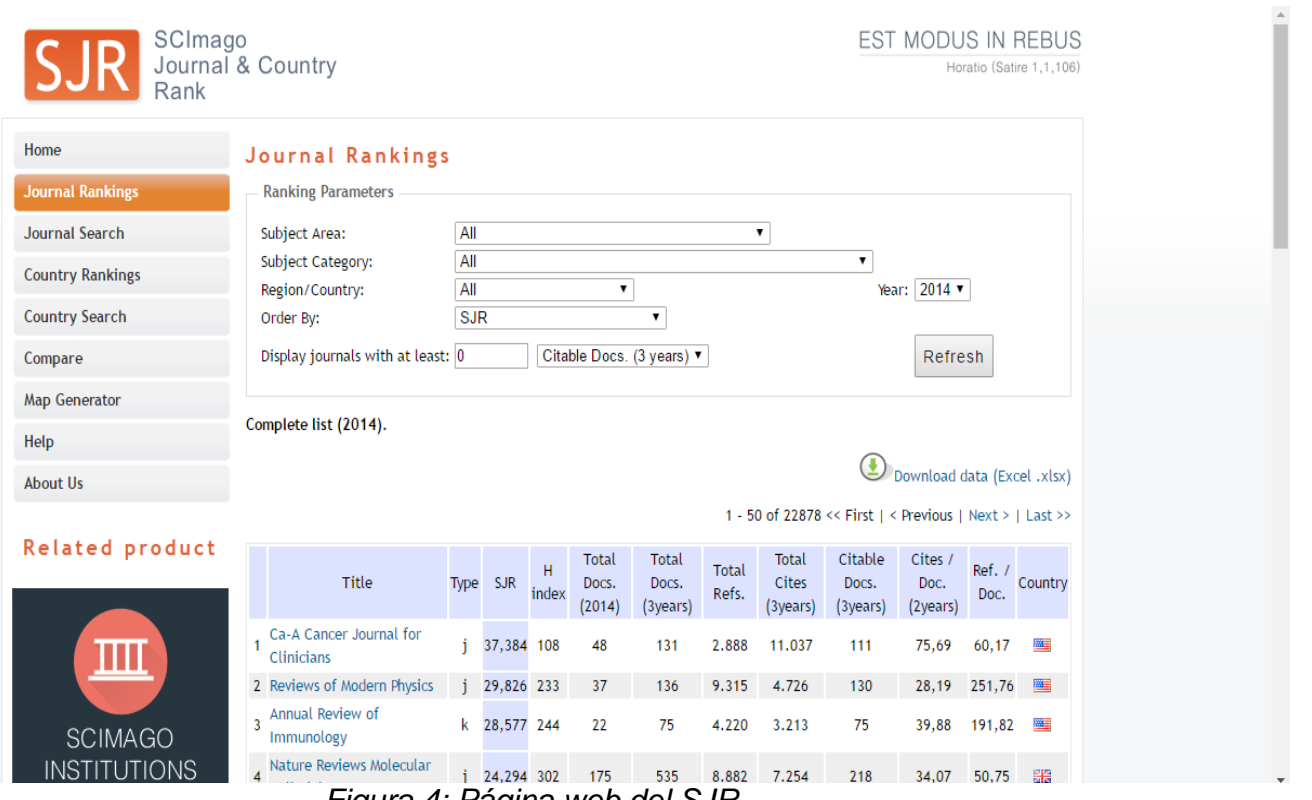

Figura 4: Página web del SJR

Por último, otro de los aspectos a tener en cuenta a la hora de publicar ya que muchas revistas empiezan a solicitarlo es el Identificador Único. En el momento en el que nos encontramos donde las publicaciones son globales nos encontramos con la problemática de que a veces errores o confusiones (incluso en otros países cambio en el apellido por matrimonio) con los nombres identificativos de los investigadores. Para evitar este problema se ha creado el denominado Open Researcher y Contributor ID (ORCID), una organización sin ánimo de lucro, internacional, abierta e interdisciplinar. Consiste en otorgar un identificador único a un investigador con el que poder reconocerlo en todos sus trabajos de una forma sencilla (Fenner y Haak, 2014).

\section{EL ANÁLISIS DE DATOS}

Sin duda uno de los momentos más especiales en cualquier proceso de investigación, es ese momento en el que el investigador comienza el proceso de análisis de datos. Una sensación de complejidad que se une a la emoción de comenzar a vislumbrar algunas respuestas a tus objetivos de investigación.

El análisis de datos no suele ser un proceso lineal, ni tan siquiera puede decirse que sea un momento exclusivamente puntual dentro del proceso de investigación, aunque claro está lo situamos ordenado en nuestros cronogramas, teniendo mayor protagonismo tras la recogida de los datos, pero que aparece siempre en el horizonte de nuestro camino investigador como una cuestión transversal desde el propio diseño de investigación.

Rodríguez, Gil y García definen este laborioso proceso como el "conjunto de manipulaciones, transformaciones, operaciones, reflexiones y comprobaciones que realizamos sobre los datos con el fin de extraer significado relevante en relación a un problema de investigación" (1996, p. 197). Es este conjunto de acciones lo que hace que esta fase se caracterice principalmente por un diálogo continuo entre la teoría y los datos obtenidos adquiriendo esa dimensión teórica - práctica.

No es asunto de este artículo entrar en la disquisición entre técnicas de análisis cuantitativos o cualitativos, estando ese debate lo suficientemente contrastado a día de hoy por numerosos autores (Creswell, Plano Clark, Guttman y Hanson, 2003; Mertens 2012; Grinnel y Unrau, 2005 y Hernández y 2014). Además, debemos tener en cuenta que la elección de la técnica dependerá principalmente del tipo de datos de los que disponemos y del tipo de análisis apropiado para lograr los objetivos de nuestra investigación. 
Dentro del análisis de datos también contamos con numerosas herramientas informáticas que nos pueden ayudar en este proceso. Muchas de estas herramientas están disponibles desde las plataformas virtuales de las Universidades, o desde los servicios de apoyo a la investigación, otras son de pago por lo que debemos analizar muy bien las ventajas y desventajas a la hora de decantarnos por una u otra herramienta. También debemos tener en cuenta que en los últimos años han proliferado las plataformas que ofrecen herramientas de análisis de datos basadas en software libre, aspecto que creemos que va ligado a los que anteriormente hemos hecho referencia como el de Open Conten investigación aumentada.

A continuación, os mostramos algunas de estas herramientas, quizás las más conocidas pero que nos parecía interesante destacar dada su fiabilidad.

Herramientas de análisis de datos cuantitativos:

- SPSS (Statistical Product and Service Solutions) es una herramienta de tratamiento de datos y análisis estadístico propiedad de IBM siendo un software comercial. Con este programa podremos realizar múltiples análisis comenzando por las característicos descriptivas de la muestra (frecuencia y descriptivos, análisis de variables categóricas: tablas de contingencia), métodos explicativos (contrastes sobre medias: procedimientos Medias y Prueba t) análisis de varianza de un factor, ANOVA de un factor, análisis de correlación lineal (bivariadas), análisis de varianza factorial y análisis de covarianza (Modelo Lineal General Univariante, métodos descriptivos (Análisis de conglomerados; procedimiento de K-medias) y otros análisis como puede ser el análisis de variables de respuesta múltiple. También debemos tener en cuenta que si necesitamos realizar un Análisis Confirmatorio deberemos acudir a otro programa, como al que hacemos referencia a continuación, ya que SPPS no trabaja con ecuaciones estructurales.

- "R" es un lenguaje y entorno de programación creado en 1993 por Ross lhaka y Robert Gentleman del Departamento de Estadística de la Universidad de Auckland, con la particularidad de que es un software GNU (General Public Licenc) es decir de uso libre. En la página principal del proyecto "R" (https://www.r-project.org) nos podemos descargar sus diferentes versiones y consultar sus manuales de uso, además de algunos libros muy interesantes y completos sobre el programa. Para muchos usuarios y autores ningún otro programa "reúne las condiciones de madurez, cantidad de recursos y manejabilidad que posee R" (Contreras, Molina y Arteaga, 2010, 2).

Herramientas de análisis de datos cualitativos:

En este caso es muy interesante explorar la página de "CAQDAS Project" (Computer Assisted Qualitative Data Analysis Software) en ella podemos encontrar un listado de enlaces a programas de análisis cualitativo, así como espacios para seminarios de formación gratuitos.

También en el caso de estas herramientas debemos tener claro que existen diferentes clasificaciones según las funcionalidades de cada uno de ellos; sistemas de codificación y recuperación, sistemas basados en reglas para la construcción de teorías, sistemas basados en la Lógica, aproximación basada en índices y sistemas de redes conceptuales (Hernández y Opazo, 2010).

Algunos de estos programas de análisis cualitativo de los que nos gustaría hablar son:

- Según palabras de Chris Atchison "NVivo es más que un simple programa de análisis de datos cualitativos; es un recurso vital para la planificación, la coordinación de equipos, la revisión de literatura, el diseño de investigación, el análisis y el informe de datos". (2014, 1). Dentro de los programas de análisis cualitativo NVivo nos ofrece muchas ventajas como el manejo de multitud de formatos para el análisis de información, Word, archivos de PDF, audio, tablas de base de datos, hojas de cálculo, 
vídeos, fotos y datos web. Otra de las ventajas que puede resultar muy útil en nuestro proceso de investigación es la posibilidad de intercambiar información de NVivo con otras aplicaciones como Micrsoft WORD y Excel, SPSS, Survey Monkey, EndNote, Evernote y OneNote. Este software es comercial, aunque podemos disponer de una versión de prueba de 14 días de duración tanto para Windows como para Mac.

- RQDA es una aplicación especifica para el análisis de datos cualitativos que esta basada en "R" y liberada bajo Licencia BSD (Berkeley Software Distribution) de software libre. Funciona en Windows, Linux / FreeBSD y las plataformas Mac OSX. Aunque comparándola con otros programas comerciales como NVivo vemos que tiene un mayor número de limitaciones ya que solamente trabaja con texto plano.

A pesar de la multitud de recursos que podamos encontrar dentro de este apartado no debemos olvidar que la elección de los mismos variará dependiendo siempre de varios factores como son, los objetivos de nuestra propia investigación, la posibilidad que nuestro Centro de Adscripción ya disponga de alguna Licencia de uso un programa de análisis, de nuestra posición frente al uso de software libres, etc., por lo que es importante no dejar este tipo de elecciones para el último momento, lo que sin duda alguna puede hacer que te precipites en la elección de la herramienta.

\section{DISCUSIÓN}

Todo lo expuesto en este artículo intenta mostrar algunos aspectos que a nuestro juicio pueden ser claves cuando emprendemos el camino de la investigación, así como algunas herramientas que nos pueden ayudar a que este proceso sea algo más "sencillo". La necesidad de ir incorporando el concepto de e-research, según la idea de Wouters y Beaullieu (2006), obliga a replantearse el proceso formativo de los investigadores noveles. Según nuestra propia experiencia y las conversaciones mantenidas con otros investigadores noveles, en muchos de los congresos a los que hemos asistido, la formación que se recibe en relación a estas herramientas es escasa, y en algunas ocasiones es puramente anecdótica. Si bien es cierto que la creación de una Tesis, de un TFM o TFG se entiende como un proceso en el que debe primar la autorregulación, para conseguir que se desarrolle una verdadera investigación aumentada (Peña, 2013), además es necesario que las instituciones pertinentes desarrollen formación específica que muestre a los alumnos el potencial de integrar este tipo de herramientas en los procesos de investigación y que nos ayuden a mantener una actitud 2.0.

Aunque es cierto que estas herramientas nos ayudan, entre otras cosas, a aliviar tareas complejas o rutinarias, debemos tener en cuenta que muchas de ellas contribuyen a ese lado "más gris" de la investigación. Desde nuestro punto de vista el movimiento open content y la necesidad de una ciencia abierta está en oposición permanente a muchas herramientas pensadas para un modelo clásico y elitista de acceso a la investigación. Actualmente nos encontramos con el doble juego que hacen algunas empresas, amparadas por el sistema actual investigación, las llamadas revistas de impacto (JCR y SJR entre otros), un juego perverso en el que nos vemos obligados a participar, y que sin duda es un negocio muy rentable que parece no estar muy interesado en esta nuevas corrientes mucho más flexibles y accesibles.

Somos conscientes que existen aún muchas cosas que mejorar en nuestros procesos de investigación y sobre todo en lo que respecta a la difusión con respecto a la industria de las publicaciones científicas. Creemos necesario que se abra un debate sobre la necesidad de una ciencia abierta, en la que el conjunto de instituciones públicas tengan garantizado el acceso a los avances de la ciencia dentro de su campo y la posibilidad de estandarizar en todos los países un sistema de publicación de acceso abierto a las investigaciones financiadas con fondos públicos.

A pesar de todo esto, como decíamos en la introducción de este artículo, nuestro objetivo fundamental en este trabajo es alentar a los futuros investigadores noveles, así como mostrar un panorama actual de algunas herramientas clave que nos pueden facilitar todo el proceso de investigación. Esperamos que algunos de los recursos presentados, que a nosotros nos han sido de total utilidad, ayuden a futuros investigadores a realizar sus proyectos de investigación. Pero 
asumimos que a nadie le podemos ahorrar las diferentes emociones que el camino investigador te proporciona; desde la angustia frente a lo que parecen cantidades inasumibles de información, la emoción ante los desafíos planteados tras las reuniones con tu director o directora de Tesis, la soledad cuando te ves obligado a encerrarte a escribir, el hastío cuando parece que nunca llegarás al final, o el vértigo de los últimos días antes de la defensa. Nosotros de momento, desconocemos que emoción es la que nos espera al final del camino.

\section{REFERENCIAS BIBLIOGRÁFICAS}

Arbeláez Gómez, M. C. (2014). Las tecnologías de la información y la comunicación (TIC) un instrumento para la investigación. Revista Investigaciones Andina, 16(29), 997-1000. Recuperado de http://www.scielo.org.co/pdf/inan/v16n29/v16n29a01.pdf

Beaulieu, A. y Wouters, P. (2008), "E-research as intervention", en Jankowski, N. (Ed.). eResearch: Transformations in Scholarly Practice (pp. 54-69). Oxon: Routledge. Recuperado de http://www.virtualknowledgestudio.nl/documents/ annebeaulieu/Eresearch\%20as\%20Intervention.pdf

Castañeda, L. y Adell, J. (2013). La anatomía de los PLEs. En L. Castañeda y J. Adell (Eds.), Entornos Personales de Aprendizaje: Claves para el ecosistema educativo en red (pp. 11-27). Alcoy: Marfil.

Contreras, J.M., Molina, E. y Arteaga, C. (2010). Introducción a la programación estadística con R para Profesores. Universidad de Granada, Grupo de Educación Estadística. Recueprado de http://www.ugr.es/ batanero/pages/ARTICULOS/libroR.pdf

Corredor Acosta, A., y Sandino León, M. (2009). Las TIC`s como herramienta de investigación científica. Revista Gondola, 4(1), 25-29.

Creswell, J. W., Plano Clark, V. L., Guttman, M. L. y Hanson, W. (2003). Advanced mixed methods research designs. En A. Tashakkori y C. Teddlie (Eds.), Handbook on mixed methods in the behavioral and social sciences (pp. 209-240). Thousand Oaks, CA, EE. UU.: SAGE

Del Cura González, M. I., y Sánchez-Celaya del Pozo, M. (2007). Hablemos de... Herramientas de búsqueda bibliográfica. GH Continuada, 6(3), 151-155. Recuperado de http://aeeh.es/wpcontent/uploads/2012/05/v6n3a416pdf001.pdf

Duarte-García, E. (2007). Gestores personales de bases de datos de referencias bibliográficas: características y estudio comparativo. El Profesional de La Informacion, 16(6), 647-656. http://doi.org/10.3145/epi.2007.nov.12

Estalella, A. Ardévol, E. (2011). e-research: desafíos y oportunidades para las ciencias sociales. Convergencia, vol. 18 ( $n$ ㅇ5) pp. 87-111. Universidad Autónoma del Estado de México. Recuperado de http://cccd.es/wp/wp-content/uploads/2012/05/10515210004.pdf

Fenner, M. (2014). Altmetrics and Other Novel Measures for Scientific Impact. In S. Bartling y S. Friesike (Eds.), Opening Science: The Evolving Guide on How the Internet is Changing Research, Collaboration and Scholarly Publishing (pp. 179-189). Cham: Springer International Publishing. http://doi.org/10.1007/978-3-319-00026-8 12

Fenner, M., Scheliga, K., y Bartling, S. (2014). Reference Management. In S. Bartling y S. Friesike (Eds.), Opening Science: The Evolving Guide on How the Internet is Changing Research, Collaboration and Scholarly Publishing (pp. 125-137). Cham: Springer International Publishing. http://doi.org/10.1007/978-3-319-00026-8 8 
Fenner, M., y Haak, L. (2014). Unique Identifiers for Researchers. In S. Bartling y S. Friesike (Eds.), Opening Science: The Evolving Guide on How the Internet is Changing Research, Collaboration and Scholarly Publishing (pp. 293-296). Cham: Springer International Publishing. http://doi.org/10.1007/978-3-319-00026-8 21

Ferreira González, I., Urrútia, G., y Alonso-Coello, P. (2011). Systematic reviews and metaanalysis: scientific rationale and interpretation. Revista española de cardiología, 64(8), 68896. http://doi.org/10.1016/j.recesp.2011.03.029

Formanchuck, A. (2013). Cultura 2.0: Nuevos modelos de comunicar, liderar y gestionar la empresa. Recuperado de https://www.youtube.com/watch?v=GMU5JiLTg7Y

Grinnell, R. M. y Unrau, Y. A. (2005). Social work: Research and evaluation: Quantitative and qualitative approaches (7a. ed.). Nueva York, NY: Oxford University Press

Gutiérrez, I. (2011). Competencias del profesorado universitario en relación al uso de tecnologías de la información y la comunicación: Análisis de la situación en España y propuesta de un modelo de formación. (Tesis Doctoral no publicada). Universidad Rovira i Virgili: Tarragona. Recuperado de http://www.tdx.cat/handle/10803/52835

Hernández Castilla, R. y Opazo Carvajal, H. (2010). Apuntes de Análisis Cualitativo en Educación. Recuperado http://www.uam.es/personal pdi/stmaria/jmurillo/Met Inves Avan/Materiales/Apuntes Cualitat ivo.pdf

Hernández, R.; Fernández, C. Y Baptista, P. (2014). Metodología de la Investigación (6ª Edic). México: McGrall Hill.

Larrosa, J. (2007). Acerca de la experiencia. Encuentro Nacional "Formar en futuro presente". Instituto Nacional de Formación Docente. Mar de Plata: Argentina.

Mertens, D. M. (2012). What comes first? The paradigm or the approach? Journal of Mixed Methods Research, 6(4), 255-257. Recuperado de http://mmr.sagepub.com/content/6/4/255.full.pdf+html

Peña, I. (2013). El PLE de investigación - docencia: el aprendizaje como enseñanza. En L. Castañeda y J. Adell (Eds.), Entornos Personales de Aprendizaje: Claves para el ecosistema educativo en red (pp. 93-110). Alcoy: Marfil.

Roberts, K. A., y Wilson, R. W. (2002). ICT and the Research Process: Issues around the Compatibility of Technology with Qualitative Data Analysis. Forum Qualitative Sozialforschung/Forum: Qualitative Social Research, 3(2). http://doi.org/http://nbnresolving.de/urn:nbn:de:0114-fgs0202234

Rodera Bermúdez, AM y González Ramos, AM (2014). "Tesis 2.0: Herramientas para su diseño, Desarrollo, lectura y Difusión". En Romero Frías, E. y Sánchez González, M. (Eds.), Ciencias Sociales y Humanidades Digitales: Técnicas, Herramientas Y Experiencias de e-Investigación e Investigación en Colaboración, Capítulo 11, 255-276. La Laguna: Sociedad Latina de Comunicación Social.

Rodríguez, G., Gil, J. y García, E. (1999). Metodología de la investigación cualitativa. Málaga: Aljibe.

Sabino, C. (1998). Prólogo. Mitos y errores en la elaboración de tesis y proyectos de investigación. Por Fidias Arias. Caracas: Episteme. 
Sánchez, M. y Teruel, L. (2013). Investigar la Comunicación hoy. Revisión de políticas científicas y aportaciones metodológicas. En M. V. Mariño Árbol, T. González Hortigüela y M. Pacheco Rueda (Coords.), Simposio Internacional sobre Política Científica en Comunicación, Vol. 1, (COMUNICACIONES 1), págs. 87-102

Sureda, J., Comas, R., Oliver, M., y Guerrero, R. M. (2010). Fuentes de información bibliográfica a través de Internet para investigadores en educación. Palma de Mallorca: REDINED. Recuperado de http://www.doredin.mec.es/documentos/01220102007215.pdf

Universidad Autónoma de Barcelona. (2009). Indicadores de impacto de revistas. Recuperado de http://biblioteca.unileon.es/documentos/guia factorimpacto.pdf

Urrútia, G., y Bonfill, X. (2010). Declaración PRISMA: una propuesta para mejorar la publicación de revisiones sistemáticas y metaanálisis. Medicina Clinica, 135(11), 507-511. http://doi.org/10.1016/i.medcli.2010.01.015

Valles, M. (2001). Ventajas y desafíos del uso de programas informáticos (p.e. ATLAS.ti y MAXqda) en el análisis cualitativo. Una reflexión metodológica desde la Grounded Theory y el contexto de la investigación social española. Ponencia presentada en el Seminario sobre Investigación Avanzada Cualitativa Asistida por Ordenador, Granada: Fundación de Centros de Estudios Andaluces. Recuperado de http://public.centrodeestudiosandaluces.es/pdfs/S200105.pdf

Wouters, P. (2006), "What is the matter with e-Science? - thinking aloud about informatisation in knowledge creation". Comunicación presentada en Pantaneto Forum. Recuperado de http://www.pantaneto.co.uk/issue23/wouters.htm

Wouters, P. y Beaulieu, A. (2006), "Imagining e-science beyond computation", en Christine Hine (Ed.]. New Infrastructures for Knowledge Production: Understanding E-Science, Hershey, PA: Information Science Publishing. Recuperado de http://www.igiglobal.com/viewtitlesample.aspx?id=27286yptid=800yt=imagining\%20escience\%20beyond\%20computation

\section{INFORMACIÓN SOBRE LOS AUTORES}

\section{Víctor González Calatayud}

Universidad de Murcia

Licenciado en Pedagogía por la Universidad de Murcia. Máster en Psicología de la Educación por la misma universidad. Actualmente becario predoctoral en el departamento de Didáctica y Organización Escolar de la Universidad de Murcia y miembro del Grupo de Investigación de Tecnología Educativa (GITE). Las líneas de investigación en las que trabaja se centran en los riesgos asociados a las TIC, fundamentalmente el ciberacoso, así como los MOOC, las metodologías enriquecidas con TIC y la enseñanza e-learning.

Twitter: @Victorgoca

Researchgate: https://www.researchgate.net/profile/Victor Gonzalez Calatayud

Web GITE: http://www.um.es/gite/cms/

\section{María del Mar Román García}

Universidad de Murcia

Graduada en Educación Social por la Universidad de Murcia. Máster en Tecnología Educativa; elearning y Gestión del Conocimiento por la Universidad de las Islas Baleares. Contratada 
predoctoral en el Departamento de Didáctica y Organización Escolar de la Universidad de Murcia y miembro del Grupo de Investigación de Tecnología Educativa. Investigadora novel interesada en: Entornos Personales de Aprendizaje, Ciudadanía Digital, Desarrollo Comunitario y Participación Social mediada por Tecnología.

Twitter: @MarimarRoman

\section{$($ (c) $)$ EY-No}

Los textos publicados en esta revista están sujetos a una licencia de Reconocimiento 4.0 España de Creative Commons. Puede copiarlos, distribuirlos, comunicarlos públicamente y hacer obras derivadas siempre que reconozca los créditos de las obras (autoría, nombre de la revista, institución editora) de la manera especificada por los autores o por la revista. La licencia completa se puede consultar en:Licencia Creative Commons Atribución-NoComercial 4.0 Internacional. 